\title{
Towards Better Technical Debt Detection with NLP and Machine Learning Methods
}

\author{
Leevi Rantala \\ leevi.rantala@oulu.fi \\ University of Oulu \\ Oulu, Finland
}

\begin{abstract}
Technical debt (TD) is an economical term used to depict nonoptimal choices made in the software development process. It occurs usually when developers take shortcuts instead of following agreed upon development practices, and unchecked growth of technical debt can start to incur negative effects for software development processes.

Technical debt detection and management is mainly done manually, and this is both slow and costly way of detecting technical debt. Automatic detection would solve this issue, but even state-of-the-art tools of today do not accurately detect the appearance of technical debt. Therefore, increasing the accuracy of automatic classification is of high importance, so that we could eliminate significant portion from the costs relating to technical debt detection.

This research aims to solve the problem in detection accuracy by bringing in together static code analysis and natural language processing. This combination of techniques will allow more accurate detection of technical debt, when compared to them being used separately from each other. Research also aims to discover themes and topics from written developer messages that can be linked to technical debt. These can help us to understand technical debt from developers' viewpoint. Finally, we will build an opensource tool/plugin that can be used to accurately detect technical debt using both static analysis and natural language processing methods.
\end{abstract}

\section{CCS CONCEPTS}

- Computing methodologies $\rightarrow$ Natural language processing; - Information systems $\rightarrow$ Clustering and classification.

\section{KEYWORDS}

natural language processing, machine learning, technical debt

\section{ACM Reference Format:}

Leevi Rantala. 2019. Towards Better Technical Debt Detection with NLP and Machine Learning Methods. In Proceedings of ICSE 2020: The 42nd International Conference on Software Engineering (ICSE '20). ACM, New York, NY, USA, 4 pages. https://doi.org/10.1145/1122445.1122456

Permission to make digital or hard copies of all or part of this work for personal or classroom use is granted without fee provided that copies are not made or distributed for profit or commercial advantage and that copies bear this notice and the full citation on the first page. Copyrights for components of this work owned by others than ACM must be honored. Abstracting with credit is permitted. To copy otherwise, or republish, to post on servers or to redistribute to lists, requires prior specific permission and/or a fee. Request permissions from permissions@acm.org.

ICSE '20, May 23-29, 2020, Seoul, South Korea

(C) 2019 Association for Computing Machinery.

ACM ISBN 978-x-xxxx-xxxx-x/YY/MM...\$15.00

https://doi.org/10.1145/1122445.1122456

\section{INTRODUCTION}

Technical debt (TD) is an economical term first introduced in the 1990's by Cunningham, and it is used to depict non-optimal choices made in the software development process [7]. Technical debt occurs usually when developers take shortcuts instead of following agreed upon development practices [1]. Unchecked growth of technical debt can yield extra development costs later in the development processes [8]. Financial costs are not the only negative aspects associated with this growth, as it can lead to weakened morale, lower productivity, worse quality, decreased code maintainability, extra costs and working hours, lack of motivation, impairing development of new features, making it harder for newcomers to comprehend the code and overall higher risks [29,32].

With all the negative effects that TD has on software development, it is worth looking at how commonly it is encountered and how long TD items can stay in projects. Recent study [25] analyzed 33 projects from Apache to discover how diffused technical debt really is. The results show, that technical debt is commonly seen in software projects. Another study [30] shows that $80 \%$ of technical debt is never removed from the developed systems, and that only $9 \%$ gets actually removed through refactoring.

TD can therefore be seen as a widespread phenomenon, and it can effect software projects for a long time. There is clearly a need for TD management, but currently there are number of issues relating to the management costs and TD identification. It has been shown that major costs for technical debt management stem from manual analysis and evaluation processes[15, 33]. Manually labeling TD items is expensive and automating these steps would be important, but even the state-of-the-art tools today do not necessarily capture from code metrics the most important parts that should be refactored [18].

This research aims to tackle this problem by bridging free-form natural language analysis and static code analysis together. The main contributions are development of a tool to automatically and accurately detect TD. We also analyze developers' written messages to uncover reasons and activities that are responsible for the appearance of technical debt.

\section{RELATED WORK}

The research is based on previous works that deal with technical debt management, its automatic detection, and static metrics relating to it. We also rely on a specific subclass of technical debt coined self-admitted technical debt, which is mainly researched with NLP techniques.

2.0.1 Technical debt management. It has been shown that major costs for technical debt management stem from manual analysis 
and evaluation processes [15]. Manual identification was used also in another study [33], where developers spent 10 weeks looking for items that could be labeled as technical debt. Manually labeling TD items is error-prone and expensive way of working, which requires several persons cross-checking and validating each others labeling for errors. Automating these steps would eliminate a significant portion of workload from TD management.

\subsection{Automatic technical debt detection}

Technical debt management would benefit greatly from automatic TD detection, but automating these analyses and evaluations is still hard. Even the state-of-the-art tools today do not necessarily capture from code metrics the most important parts that should be refactored. One study [18] explored SonarQube's metrics and discovered that from the over 200 violations that are listed in the tool's manual, only 26 have a low possibility for causing faults, and that code labeled as a bug practically never led to actual errors. Authors note that SonarQube's estimations do not accurately reflect the real amount of work needed for refactoring and maintenance, nor do they portray accurately the actual costs related to these activities.

\subsection{Self-admitted technical debt}

Self-admitted technical debt (SATD) is technical debt, which is accompanied with a message from the developer admitting that debt was taken. Previous studies have researched SATD by analyzing source code comments [16, 24], analyzing diffusion and history of code as well as commit message analysis relying on identification of manually selected keywords [31]. Another study investigated whether technical debt is discussed at issue trackers [4]. Their findings show that sometimes developers do use technical debt and related concepts, but this is very rare.

\subsection{Static technical debt metrics}

One possibility for technical debt detection is to utilize metrics which are based on code smells and object-oriented programming.

Code smells indicate poor code quality, and are therefore closely related to TD. The 22 smells introduced in [3] and their taxonomies from [22] can be utilized for technical debt detection/classification. Exact thresholds for code smells can be hard to decide, but previous research offers some guidelines on manually decided thresholds [10] or levels based on machine-learning [13]. Object-oriented metrics such as the ones proposed by Chidamber and Kemerer [6] and later supplemented by $\mathrm{Li}[20]$ can be used in conjunction with the code smell metrics. Lastly, we can use classifications and metrics from existing tools, as was done in [12].

It is important to note, that code based detection can only reveal part of the TD in the system [17]. Therefore it is important to combine these metrics with other techniques such as NLP analysis, as TD can exist even when it doesn't violate any coding guidelines.

\section{RESEARCH HYPOTHESES}

The aim of this research is to combine NLP with static code analyses in order to develop new methods to better detect and analyze technical debt. This is done by investigating the written communications (commit messages, code comments, issue tracker messages etc.) of the developers in relation to accumulation of TD. We enhance TD detection by creating new models and resources that help in detecting technical debt from textual messages and code metrics. The investigation into the written communications include analyzing what kind of themes and topics emerge from these communications, and how these communications can be mapped to code level changes. Finally we build a tool to both detect appearance of technical debt as well as reporting developer communication patterns. These aims are achieved through following hypotheses:

Hypothesis 1 - Employing automated NLP techniques rather than relying on manually chosen predictor terms, or phrases leads to higher accuracy in technical debt detection. The first hypothesis claims that automating NLP techniques to detect patterns predicting technical debt can lead to better results than relying on manually created NLP resources. Previous studies have examined technical debt through NLP by looking at manually chosen and labeled predictor terms [31] from commit messages, or phrases [24] on code level commits.

A previous study [31] looked at if a commit message contained one of five manually chosen predictor terms that were: refactor, bug, feature, improve, and document. They report an area under curve (AUC) of 0.57 , when predicting the appearance of SATD only through comments messages. In our first and yet unpublished study, we show that selecting automatically words instead of relying on the appearance of manually chosen ones can lead to higher performance when using commit messages to technical debt detection.

Instead of commit messages, NLP can be applied to code level comments for technical debt detection. In a previous study [16], it was shown that relying on manually created phrases [24] for technical debt does not guarantee best results. In our second and ongoing study, we are building a classifier that can predict when a developer should have labeled a code comment to contain "TODO"keyword. This word is important, as state-of-the-art tools such as SonarQube [26] rely on detecting individual keywords. This causes problems in detection accuracy, as some of the relevant comments are missed due to a missing keyword. Therefore, identifying automatically comments which should have such a keyword can improve their performance.

Hypothesis 2 - NLP analysis reveals topics of developers' written messages when they have committed technical debt. We predict that there will be a connection on the messages the developers write, and the appearance of certain kind of technical debt. This can mean that they use certain types of expressions or words. As an example, we can look at actual SATD-comments labeled in a previous study [21]:

- Example 1: TODO we should have better logging than stderr

- Example 2: TODO: Handle empty contents

- Example 3: TODO: Work on a better way for customizing keybindings

- Example 4: TODO: Maybe integrate this with PaddingManager.

- Example 5: TODO This should ideally be using it's own class (instead of ClientRMSecurityInfo)

- Example 6: TODO should be conf injected?

Examples 1 and 3 talk about non-optimal code, which indicates that there should be a better implementation than the one used. Example 2 is about a missing functionality, which should be taken 
into account. Examples 4 and 5 speak about performing refactoring. Finally the last one expresses uncertainty about the implemented code, and if it should be changed or not.

Our prediction is that we can identify certain topics such as "nonoptimal implementation" in the Examples 1 \& 3 above, "refactoring" in Examples 4 \& 5, and "uncertainty" in Example 6.

In a way, our work is similar to [9], where the authors classified SATD comment to different TD types. They identified 5 different categories: design, defect, documentation, requirement and testing debt. We are not looking at TD classification, but rather what the topics are being expressed / discussed. In this way we are complementing the work done by [9]. Combining their approach and ours, we could label the Example 6 from above as "Design debt, Uncertain Developer", where Design Debt is classification from [9], and Refactoring is the topic we identified from the comment.

The technical details on how this topic identification and classification is accomplished are not yet set in stone, but one possibility is to use Latent Dirichlet Allocation (LDA) for clustering words to topics [5]. LDA is a versatile technique, which has been used previously e.g. to prioritize test cases [28], examine code evolution [27] and analyze developer discussions on Stack Overflow [2]. LDA relies on three critical parameters: number of topics, and hyper parameters $\alpha$ and $\beta$ which define how many topics one text has, and how many words are in one topic. Deciding these and especially the number of topics is critical for LDA performance [14]. One possibility is to use a genetic algorithm such a DEOptim ${ }^{1}$ to find best parameters by optimizing rate of perplexity change (RPC) [34].

Using results from LDA analyses, we can build a model which can analyze code level messages and label them with different topics. This helps to monitor the system's status and to identify what kind of actions are needed in different parts of the system.

The textual data does not need to be only from code level, and it could be combined from a number of different sources including issue trackers, commit messages, and other sources. Issue trackers have especially been shown to be one possible place where developers talk about technical debt [11], and that utilizing machine learning tools to discover technical debt items should be investigated further [4].

Hypothesis 3 - There exists a connection between identified topics on written messages and code level changes. We hypothesize that we can map the topics discovered in Hypothesis 2 to certain types of changes on the code level by combining NLP results to other software metrics. One example is that when we identify topics such as "Refactoring Needed" or "Non-optimal code" from Examples 3 and 5 above, we will also analyze the code for metrics. Maybe in these cases the results could be "Non-optimal Code, Developing to Long Method", "Refactoring Needed, Developing to Feature Envy". Based on millions of analyses, we can make general and projectbased predictions such as: "Uncertainty in Development leads in $65 \%$ cases to Long Method, 30\% cases to God Class and 5\% has no apparent TD."

This also works the other way, so that we can pinpoint from code metrics possible weak spots on the system, which do not have yet a comment. Analyzing millions of code comments and metrics, we can then possibly propose what kind of comment there should be

\footnotetext{
${ }^{1}$ https://cran.r-project.org/web/packages/DEoptim/index.html
}

injected. Previous research [11] lists "lack of code documentation" as one of the top-3 causes for technical debt. Linking the discovered topics from our second hypotheses to different software metrics and vice versa, we can make technical debt items and their nature more visible.

Hypothesis 4 - Using NLP methods to identify appearance of technical debt can be utilized in the creation of a tool that helps developers to better identify when debt has been created. With the experiences gained from Hypotheses 1 - 3, we will create an independent tool or a plugin to detect technical debt with NLP methods. Utilizing NLP in conjunction with other methods has been shown to improve the results, and sometimes offering much better cost-effectiveness [31].

The technical details of the tool are still open, but it most likely will be created both as an individually usable module for programming purposes, as well as an plugin to an existing popular IDE such as Eclipse.

\section{RESEARCH METHODS AND MATERIALS}

Research will be based on mining software repositories and issue trackers for data, which is then analyzed using NLP methods and machine learning implementations. Software repository mining is an efficient way to gather large amounts of data for analysis, and it has been utilized in previous studies [23, 24, 31]. Our study will mine and examine several open-source repositories from different projects. Utilizing several projects from different software domains will not only increase the size of the data set, but it can reveal similarities and differences between developers and projects. One possibility is also to use an already created software repository dataset, such as Technical Debt Dataset [19], which guarantees more easily reproducible results for other researchers.

In this research repository mining will not only include developers' comments, but also different metrics from commits to identify technical debt from the code using NLP methods. These metrics include, but are not limited to, number of lines added, deleted or changed per commit, and different technical debt metrics and their changes at commit level granularity such as several method complexity metrics. The NLP methods of this research include utilizing different methods such as bag-of-words (BoW), Latent Dirichlet Allocation (LDA) and word embeddings (WE). These can be supplemented with other techniques such as dependency parsing and named-entity recognition. Utilizing aforementioned NLP methods, we will create different open-source resources that can be utilized on their own or part of some analysis tool. These resources include, but are not limited to activity and topic lexicons, and a visualization plugin for an existing tool. The lexicons offer an inexpensive and relatively easy way to train and evaluate different classifiers that analyze textual data around technical debt.

In addition, we are in contact with a company partner during the research in order to test our methodology in an industry environment.

\section{EXPECTED CONTRIBUTIONS}

The contributions of the studies performed during the $\mathrm{PhD}$ thesis can be summarized broadly as follows: 
- Developing new methods for technical debt detection from developers code comments, commit messages, issue trackers and other written sources

- Creation of open-source NLP resources to aid in further development of toold for technical debt detection

- Looking at different themes and patterns that revolve around technical debt from written messages

- Designing a method to tie the identified patterns to code level changes, to enhance technical debt detection

- Developing a tool/plugin to help detecting technical debt introduction by analyzing both NLP and code metrics

The main contributions of this work are the development of the new tool/plugin to better detect technical debt by analyzing textual messages with NLP, and looking deeper into the developers messages connected to technical debt. The tool/plugin can be used either individually or in combination with other tools/plugins to detect technical debt better by complementing each other. The NLP resources, such as lexicons, will be offered as open-source online resources, to be used in future research. The themes and topics discovered by analyzing the messages can yield additional information, and possibly offer a completely new venue from which to investigate technical debt. Social aspects such as different written communications are an area that has not been yet fully utilized in relation to technical debt.

We conduct our research in an open way, and release all our datasets, codes and developed tools as open-source in order to benefit the research community and the industry.

\section{COMPLETION TIMELINE}

The author is a 1st year $\mathrm{PhD}$ student, who has completed the first full journal paper regarding Hypotheses 1 . The paper is still in review. Work for a second paper regarding "TODO"-keyword prediction is currently underway, and it will be submitted to a conference, such as SANER or MSR. Work relating to Hypotheses $2 \& 3$ will begin after completing the second paper. The expected timeline for papers to be published from them will be within 2020/1. The tool/plugin will be published in 2022, which is also when the student is expected to defend his thesis.

\section{REFERENCES}

[1] Eric Allman. 2012. Managing technical debt. Commun. ACM 55, 5 (2012), 50-55.

[2] Anton Barua, Stephen W Thomas, and Ahmed E Hassan. 2014. What are developers talking about? an analysis of topics and trends in stack overflow. Empirical Software Engineering 19, 3 (2014), 619-654.

[3] Kent Beck, Martin Fowler, and Grandma Beck. 1999. Bad smells in code. Refactoring: Improving the design of existing code 1 (1999), 75-88.

[4] Stephany Bellomo, Robert L Nord, Ipek Ozkaya, and Mary Popeck. 2016. Got technical debt?: surfacing elusive technical debt in issue trackers. In Proceedings of the 13th International Conference on Mining Software Repositories. ACM, 327-338.

[5] David M Blei, Andrew Y Ng, and Michael I Jordan. 2003. Latent dirichlet allocation. fournal of machine Learning research 3, Jan (2003), 993-1022.

[6] Shyam R Chidamber and Chris F Kemerer. 1994. A metrics suite for object oriented design. IEEE Transactions on software engineering 20, 6 (1994), 476-493.

[7] Ward Cunningham. 1992. The WyCash portfolio management system, Addendum to the proceedings on Object-oriented programming systems, languages, and applications (Addendum)

[8] Bill Curtis, Jay Sappidi, and Alexandra Szynkarski. 2012. Estimating the principal of an application's technical debt. IEEE software 29, 6 (2012), 34-42.

[9] Everton da Silva Maldonado, Emad Shihab, and Nikolaos Tsantalis. 2017. Using natural language processing to automatically detect self-admitted technical debt. IEEE Transactions on Software Engineering 43, 11 (2017), 1044-1062.
[10] Robert J Eisenberg. 2012. A threshold based approach to technical debt. ACM SIGSOFT Software Engineering Notes 37, 2 (2012), 1-6.

[11] Neil A Ernst, Stephany Bellomo, Ipek Ozkaya, Robert L Nord, and Ian Gorton. 2015. Measure it? manage it? ignore it? software practitioners and technical debt. In Proceedings of the 2015 10th Foint Meeting on Foundations of Software Engineering. ACM, 50-60.

[12] Francesca Arcelli Fontana, Vincenzo Ferme, and Stefano Spinelli. 2012. Investigating the impact of code smells debt on quality code evaluation. In 2012 Third International Workshop on Managing Technical Debt (MTD). IEEE, 15-22.

[13] Francesca Arcelli Fontana, Mika V Mäntylä, Marco Zanoni, and Alessandro Marino. 2016. Comparing and experimenting machine learning techniques for code smell detection. Empirical Software Engineering 21, 3 (2016), 1143-1191.

[14] Ying Fu, Meng Yan, Xiaohong Zhang, Ling Xu, Dan Yang, and Jeffrey D Kymer. 2015. Automated classification of software change messages by semi-supervised Latent Dirichlet Allocation. Information and Software Technology 57 (2015), 369-377.

[15] Yuepu Guo, Rodrigo Oliveira Spínola, and Carolyn Seaman. 2016. Exploring the costs of technical debt management-a case study. Empirical Software Engineering 21, 1 (2016), 159-182.

[16] Qiao Huang, Emad Shihab, Xin Xia, David Lo, and Shanping Li. 2018. Identifying self-admitted technical debt in open source projects using text mining. Empirical Software Engineering 23, 1 (2018), 418-451.

[17] Philippe Kruchten, Robert L Nord, and Ipek Ozkaya. 2012. Technical debt: From metaphor to theory and practice. Ieee software 29, 6 (2012), 18-21.

[18] Valentina Lenarduzzi, Antonio Martini, Davide Taibi, and Damian Andrew Tamburri. 2019. Towards surgically-precise technical debt estimation: early results and research roadmap. In Proceedings of the 3rd ACM SIGSOFT International Workshop on Machine Learning Techniques for Software Quality Evaluation. ACM, 37-42.

[19] Valentina Lenarduzzi, Nyyti Saarimäki, and Davide Taibi. 2019. The technical debt dataset. In Proceedings of the Fifteenth International Conference on Predictive Models and Data Analytics in Software Engineering. ACM, 2-11.

[20] Wei Li. 1998. Another metric suite for object-oriented programming. fournal of Systems and Software 44, 2 (1998), 155-162.

[21] Everton da S Maldonado, Rabe Abdalkareem, Emad Shihab, and Alexander Serebrenik. 2017. An empirical study on the removal of self-admitted technical debt. In 2017 IEEE International Conference on Software Maintenance and Evolution (ICSME). IEEE, 238-248.

[22] Mika Mantyla, Jari Vanhanen, and Casper Lassenius. 2003. A taxonomy and an initial empirical study of bad smells in code. In International Conference on Software Maintenance, 2003. ICSM 2003. Proceedings. IEEE, 381-384.

[23] Marco Ortu, Bram Adams, Giuseppe Destefanis, Parastou Tourani, Michele Marchesi, and Roberto Tonelli. 2015. Are bullies more productive?: empirical study of affectiveness vs. issue fixing time. In Proceedings of the 12th Working Conference on Mining Software Repositories. IEEE Press, 303-313.

[24] Aniket Potdar and Emad Shihab. 2014. An exploratory study on self-admitted technical debt. In 2014 IEEE International Conference on Software Maintenance and Evolution. IEEE, 91-100.

[25] Nyyti Saarimäki, Valentina Lenarduzzi, and Davide Taibi. 2019. On the diffuseness of code technical debt in Java projects of the apache ecosystem. In Proceedings of the Second International Conference on Technical Debt. IEEE Press, 98-107.

[26] SonarQube. 2019. Rules explorer. Retrieved June 27, 2019 from https://rules. sonarsource.com/

[27] Stephen W Thomas, Bram Adams, Ahmed E Hassan, and Dorothea Blostein. 2010. Validating the use of topic models for software evolution. In 2010 10th IEEE Working Conference on Source Code Analysis and Manipulation. IEEE, 55-64.

[28] Stephen W Thomas, Hadi Hemmati, Ahmed E Hassan, and Dorothea Blostein. 2014. Static test case prioritization using topic models. Empirical Software Engineering 19, 1 (2014), 182-212.

[29] Edith Tom, AybüKe Aurum, and Richard Vidgen. 2013. An exploration of technical debt. Journal of Systems and Software 86, 6 (2013), 1498-1516.

[30] Michele Tufano, Fabio Palomba, Gabriele Bavota, Rocco Oliveto, Massimiliano Di Penta, Andrea De Lucia, and Denys Poshyvanyk. 2017. When and why your code starts to smell bad (and whether the smells go away). IEEE Transactions on Software Engineering 43, 11 (2017), 1063-1088.

[31] Meng Yan, Xin Xia, Emad Shihab, David Lo, Jianwei Yin, and Xiaohu Yang. 2018. Automating change-level self-admitted technical debt determination. IEEE Transactions on Software Engineering (2018).

[32] Jesse Yli-Huumo, Andrey Maglyas, and Kari Smolander. 2015. The benefits and consequences of workarounds in software development projects. In International Conference of Software Business. Springer, 1-16.

[33] Jesse Yli-Huumo, Andrey Maglyas, Kari Smolander, Johan Haller, and Hannu Törnroos. 2016. Developing Processes to Increase Technical Debt Visibility and Manageability-An Action Research Study in Industry. In International Conference on Product-Focused Software Process Improvement. Springer, 368-378.

[34] Weizhong Zhao, James J Chen, Roger Perkins, Zhichao Liu, Weigong Ge, Yijun Ding, and Wen Zou. 2015. A heuristic approach to determine an appropriate number of topics in topic modeling. In BMC bioinformatics, Vol. 16. Springer, S8. 